\title{
Carcinoma de células escamosas de piel. Serie de casos
}

\author{
Osvaldo Iribarren B. ${ }^{1,2}$, Muriel Ramírez S. ${ }^{2}$ PhD, Juan A. Madariaga G. ${ }^{1,2}$, \\ Óscar Riveros F. ${ }^{2}$, Claudia Valdés V. ${ }^{2}$ y Javiera Toledo S. ${ }^{2}$
}

\section{Carcinoma of squamous cells of skin. Series of cases}

Introduction: Squamous skin cancer (SSC) is the second most frequent skin cáncer, nevertheless reports about this issue are not published in Chile. Objetive: To investigate social, demographics, and clinic characteristics of SSC in semidesertic Coquimbo Region, Chile. Material and Methods: serie of patients diagnosed and treated in Coquimbo hospital between January 2006 and December 2015. Inclussion criteria: 1.- histopathological confirmation of SSC. Exclusion criteria: 1.- follow up lesser than 12 months; 2.- operated in another hospital; 3.- submitted to another treatment prior to surgery; 4.- skin metastasis of mucous squamous carcinoma; 5.- patients with distant metástasis. Independent variables: age, gender, tumor site, tumor size, clinical lymph nodes, shore or valley residency. Dependent variable: recurrency frecuence, factors of recurrency, letality. Statistical analysis: descriptive and analytical by SSPS program. Results: 2.202 skin cancer cases were registered, 1.487 basal cells carcinoma (67.5\%), 181 melanoma $(8.2 \%)$ and 534 squamous cells carcinoma (24.2\%). 236 patients with complete data are included in this report. There were 153 men (64.8\%), and 83 women $(35.2 \%)$. Mean age was 75.5 years old \pm 11.7 (range 46-94). Primary site was: head 158 patients (66.9\%), other sun exposed areas 47 patients (20\%), and non exposed areas 31 patients. Cheek and front were the most frequents head site with 40 cases $(25 \%)$ and 29 cases $(12.3 \%)$, respectively. In 119 cases $(50.4 \%)$ SCC was ulcerated, and 117 cases was not; primary tumor diameter was 22 millimeters (range 3-100). Clinical lymph nodes were primarily positives in 10 patients, (4.2\%). In 12 cases with negative lymph nodes, sentinel limph node was resected. 2 were positives. Histological borders were tumor free in 201 patients $(85.2 \%)$ and, 35 cases $(14.8 \%)$ had positive histological borders. Local recurrence incidence was $8.5 \%$ (20 cases). Limph nodes recurrence was $2.1 \%$ ( 5 cases). Cancer recurrence was associated with histological positive borders $\mathrm{P}=0.001$, IC $95 \% 3.12$ 12.19 , and ulcerated tumor $\mathrm{p}=0.01$, OR 4.63 ; IC $1.59-13.50$. Letality was $2.56 \%$. Mean follow up was 36 months (range 12-228 months). Conclusions: SSC has a good prognosis when primary tumor is resected early, with free histological borders resection.

Key words: squamous cell cancer; skin cancer; squamous skin cancer.

\section{Resumen}

Introducción: El cáncer de células escamosas (CCE) es el segundo cáncer de piel más frecuente. Sin embargo, no hay publicaciones en Chile sobre el tema. Objetivo: Investigar características sociodemográficas y clínicas del CCE en la Región de Coquimbo, Chile. Material y Métodos: Serie de casos de pacientes con diagnóstico de CCE de piel tratados en el hospital de Coquimbo, entre enero de 2006 y diciembre de 2015. Criterios de inclusión: diagnóstico histológico definitivo de CCE de piel. Criterio de exclusión: 1.- seguimiento posoperatorio menor de 12 meses; 2.- operado en otro hospital; 3.- sometido a otro tratamiento previo a la cirugía; 4.- metástasis cutáneas de un CCE mucoso; 5.- CCE con metástasis a distancia. Variables independientes: edad, género, localización, tamaño, linfonodos comprometidos, residencia costera-interior. Variables dependientes: recurrencia, factores de recurrencia, letalidad. Análisis estadístico: descriptivo y analítico con el programa SSPS. Resultados: Se registraron 2.202 casos de cáncer de piel, 1.487 basocelular (67,5\%), 181 melanomas (8,2\%) y 534 CCE (24,2\%). 236 pacientes tienen datos completos y constituyen el informe, 153 hombres $(64,8 \%)$ y 83 mujeres $(35,2 \%)$. Edad: 75,5 años $\pm 11,7$ (extremos 46-94). La localización es: cabeza 158 casos (66,9\%), otras áreas expuestas $47(20 \%)$ 
y no expuestas $31(14,1 \%)$. En cabeza la localización más frecuente es mejilla 40 casos $(25 \%)$, frente 29 casos (12,3\%). En 119 casos (50,4\%) el cáncer se presenta ulcerado y en 117, no ulcerado (49,6\%); diámetro del tumor 22 milímetros (rango 3-100 mm). En 10 casos hay linfonodos clínicos (4,2\%). En 12 casos $(5,9 \%)$ se extirpa LNC, 2 positivos. 201 casos presentan bordes histológicos libres $(85,2 \%)$ y en 35 casos, borde comprometido (14,8\%). Tasa de recurrencia local $8,5 \%$ (20 casos) y ganglionar $2,1 \%$ (5 casos). Recidiva del cáncer se asocia a borde histológico comprometido: $\mathrm{P}=0,001, \mathrm{IC} 95 \%$ 3,12-12,19 y ulceración p = 0,01, OR 4,63; IC 1,59-13,50. Seguimiento de 36 meses (rango 12-228). Letalidad 2,56\%. Conclusión: El CCE de piel extirpado precozmente con confirmación histológica de erradicación tiene buen pronóstico.

Palabras clave: cáncer de células escamosas; cáncer de piel; cáncer escamoso de piel.

\section{Introducción}

El cáncer de piel no melanoma es el cáncer más común e incluye el carcinoma de células basales y el carcinoma de células escamosas $(\mathrm{CCE})^{1}$. La base y el modelo para resolver el problema del cáncer de la piel es la cirugía ${ }^{1,2}$. Ambas enfermedades comparten el hecho que entre sus factores de riesgo más importantes se considera la exposición acumulativa a la radiación UV, la exposición a arsénico inorgánico, individuos de piel clara, piel dañada por el sol, la población mayor de 60 años e infecciones virales ${ }^{3-8}$. Comparten, además, que son localmente invasores pero, se distinguen en que el primero raramente genera metástasis y el segundo tiene ese potencial especialmente cuando se localiza en las orejas, labios y zonas cubiertas de piel, tumores que crecen en úlceras crónicas y tumores pobremente diferenciados $^{5}$. El mayor riesgo publicado de desarrollar metástasis fluctúa entre 5 y $14 \% \%^{9,10}$.

Los pilares para un control efectivo de esta enfermedad, radica en la identificación de los pacientes de riesgo, detección precoz y el tratamiento quirúrgico rápido del mismo ${ }^{11}$. Por su parte, la presencia de metástasis en los linfonodos regionales es el principal factor de mal pronóstico para la sobrevida de los enfermos ${ }^{12}$. Los predictores más importantes de metástasis en linfonodos son el número de factores de alto riesgo para la enfermedad, invasión perineural e invasión vascular ${ }^{10}$. En los pacientes con linfonodos, clínicamente negativos, se ha introducido la resección y estudio histológico del linfonodo centinela (LNC), con el objeto de mejorar el control de la enfermedad y disminuir la morbilidad de la cirugía sobre los territorios ganglionares correspondientes. En CCE de piel algunos estudios sobre LNC ha mostrado una especificidad de $100 \%$ y un valor predictivo negativo de $96 \%{ }^{13}$, pero otros no respaldan su capacidad para identificar a los pacientes, con linfonodos clínicamente negativos y, que evolucionarán con metástasis a lo largo de su seguimiento ${ }^{14}$.

La incidencia del CCE varía entre 104105/100.000 habitantes, en Europa Central ${ }^{15,16}$ y 120/100.000 en Brasil ${ }^{17}$. En Chile no hay datos sobre incidencia de esta enfermedad. La tasa de cáncer basocelular, en mayores de 70 años en nuestra región es de $436 \times 100.000^{18}$, tasa considerablemente alta en el contexto internacional, asociada a alta radiación ultravioleta ambiental. Asumimos, por lo tanto, que la incidencia regional de CEE de piel también debe tener valores elevados.

El objetivo de este estudio es investigar las características sociodemográficas y clínicas de portadores de CCE de piel, de acuerdo con el género, edad, lugar de residencia, tipo y localización del cáncer, incidencia, factores de recurrencia luego del tratamiento, desenlace del tratamiento quirúrgico y letalidad, en residentes en la Región de Coquimbo (Chile), un área con clima semidesértico árido, caracterizado por alta radiación ultravioleta.

\section{Material y Métodos}

\section{Tipo de estudio: Serie de casos retrospectivo}

Muestra: no probabilística de todos los pacientes con el diagnóstico histológico confirmado de $\mathrm{CCE}$ de piel ingresados en el Servicio de Patología del Hospital San Pablo de Coquimbo, entre enero de 2005 y diciembre de 2014.

Criterios de inclusión: pacientes con diagnóstico histológico definitivo de CCE de piel.

Criterio de exclusión: 1) seguimiento posoperatorio menor de 12 meses; 2) operado en otro hospital; 3) sometido a otro tratamiento previo a la cirugía; 4) metástasis cutáneas de un CCE mucoso; 5) CCE con metástasis a distancia. 


\section{Recolección de datos: planilla Excel}

Variables independientes: edad, género, localización del tumor, tamaño el tumor, linfonodos comprometidos, residencia costera-interior, actividad.

Variables dependientes: intervalo libre de enfermedad, recurrencia, letalidad, sobrevivencia.

Los tumores se clasifican según las características de riesgo de progresión de la enfermedad, en alto y bajo riesgo ${ }^{19}$. Este modo de clasificar los cánceres de piel se sustenta en la $7^{\mathrm{a}}$ edición de la clasificación de tumores malignos de la American Join Comittee on Cancer de 2010 ${ }^{20}$. Los tumores de alto riesgo incluyen alto grado histológico, invasión tumoral subcutánea, invasión perineural, tumores recurrentes y localización de alto riesgo: oreja, labio, sitio no expuesto al sol, aparición en úlcera crónica o cicatriz previa y tumor de más de $20 \mathrm{~mm}$ de diámetro ${ }^{3,19,20}$. Se considera de bajo riesgo aquellos que no presentan esos atributos. Los tumores de bajo grado se resecan con un margen de seguridad de a lo menos $5 \mathrm{~mm}$ de diámetro y aquellos de alto grado se resecan con un margen macroscópico sano, mínimo de $1 \mathrm{~cm}^{19}$. Se intenta, en la mayoría de los casos, la biopsia contemporánea por congelación de rutina para asegurar la resección completa en la primera intervención ya que la resección incompleta se comporta con una recidiva significativamente mayor ${ }^{21,22}$.

Desde 2005, en los pacientes con melanoma ${ }^{23} \mathrm{y}$, más tarde, CCE de piel con linfonodos clínicos efectuamos biopsia de LNC contemporánea a la resección del tumor primario, mediante la inyección del colorante azul de isosulfán, en la dermis alrededor de la lesión primaria. En los casos de compromiso positivo del LNC se realiza, en una segunda cirugía, la resección ganglionar regional. En los pacientes con evidencia clínica y/o ecográfica de metástasis en los linfonodos regionales se realiza la resección del tumor primario y la resección de los grupos ganglionares regionales en la misma cirugía ${ }^{24}$.

Principios de reconstrucción: las características del paciente, la localización del defecto y el tamaño son los elementos considerados para escoger la opción de reconstrucción. Los defectos menores de 2 $\mathrm{cm}$ con piel laxa vecina son cerrados en forma primaria. De mayor tamaño son cerrados con colgajos locales disponibles o colgajos a distancia o injertos de piel total por su rapidez de ejecución y reducción del tiempo quirúrgico ${ }^{25}$.

Los pacientes fueron sometidos a seguimiento para pesquisa de recidiva local, diseminación regional o diseminación a distancia mediante control trimestral los primeros 2 años y semestral en los años siguientes. Los pacientes que no acudieron a control por dos años o más fueron evaluados a través de una entrevista telefónica al cierre de este estudio.

Análisis estadístico: descriptivo y analítico; se realizó con el programa SSPS para Windows. Los valores de distribución normal son expresados como media \pm desviación estándar y las variables con valores extremos son expresados como mediana y rango. Para determinar asociación entre variables cualitativas se realiza la prueba de $\chi^{2}$ y para variables cuantitativas la prueba de $t$ de Student. Se considera significativo un valor de $\mathrm{p}<0,05$.

Aspectos éticos: el diseño del estudio es retrospectivo con base en las historias clínicas; el estudio fue sometido a revisión por el Comité Ético Científico del Servicio de Salud Coquimbo el que, por resolución del 1 de abril de 2016, establece que no se encuentran reparos éticos y puede generar mayor conocimiento sobre el tema.

\section{Resultados}

En el período 2005-2014, en el Servicio de Anatomía Patológica se registraron 2.014 pacientes con diagnóstico de cáncer de piel. De ellos, 1.487 corresponden a cáncer basocelular (64\%), 181 casos de melanoma ( $8 \%$ ) y 534 pacientes con diagnóstico confirmado de CCE (25\%). En estos últimos, 17 casos se excluyeron por registrar un seguimiento menor a 1 año, 60 pacientes por datos incompletos y 128 historias clínicas desaparecieron o resultaron destruidas con ocasión del terremoto de septiembre de 2015 que destruyó el hospital. En consecuencia, el número de pacientes reportados es de 236 casos (Figura 1).

Se registran 153 hombres $(64,85)$ y 83 mujeres $(35,2)$, edad media de 75,5 años $\pm 11,7$ (38-94 años). La tasa de incidencia es $46 \times 100.000$ en valles interiores y $37,2 \times 100.000$ en habitantes de la zona costera de la región de Coquimbo. El diámetro de la lesión fue de 21,3 $\mathrm{mm} \pm 16,4(3-100 \mathrm{~mm})$. La residencia de los pacientes es costera en 131 casos $(64,8 \%)$ e interior en $83(35,2 \%)$. En 206 casos $(87,3 \%)$ el cáncer se localiza en piel expuesta y en 30 casos $(12,75)$ en piel no expuesta al sol. En 200 $(84,7 \%)$ casos hay fotodaño avanzado de la piel en el sitio del cáncer y, en 36 éste está ausente (15,3\%) porque el cáncer se ubica en área no expuesta a radiación ultravioleta (RUV). En 119 casos $(50,4 \%)$ el cáncer se presenta ulcerado y en 117 casos no ulcerado $(49,6 \%)$. En 10 casos hay linfonodos clínicos $(4,2 \%)$ (Tabla 1).

La localización es en cabeza en 158 casos $(66,9 \%)$, mano 20 casos $(8,5 \%)$, cuello 9 casos 


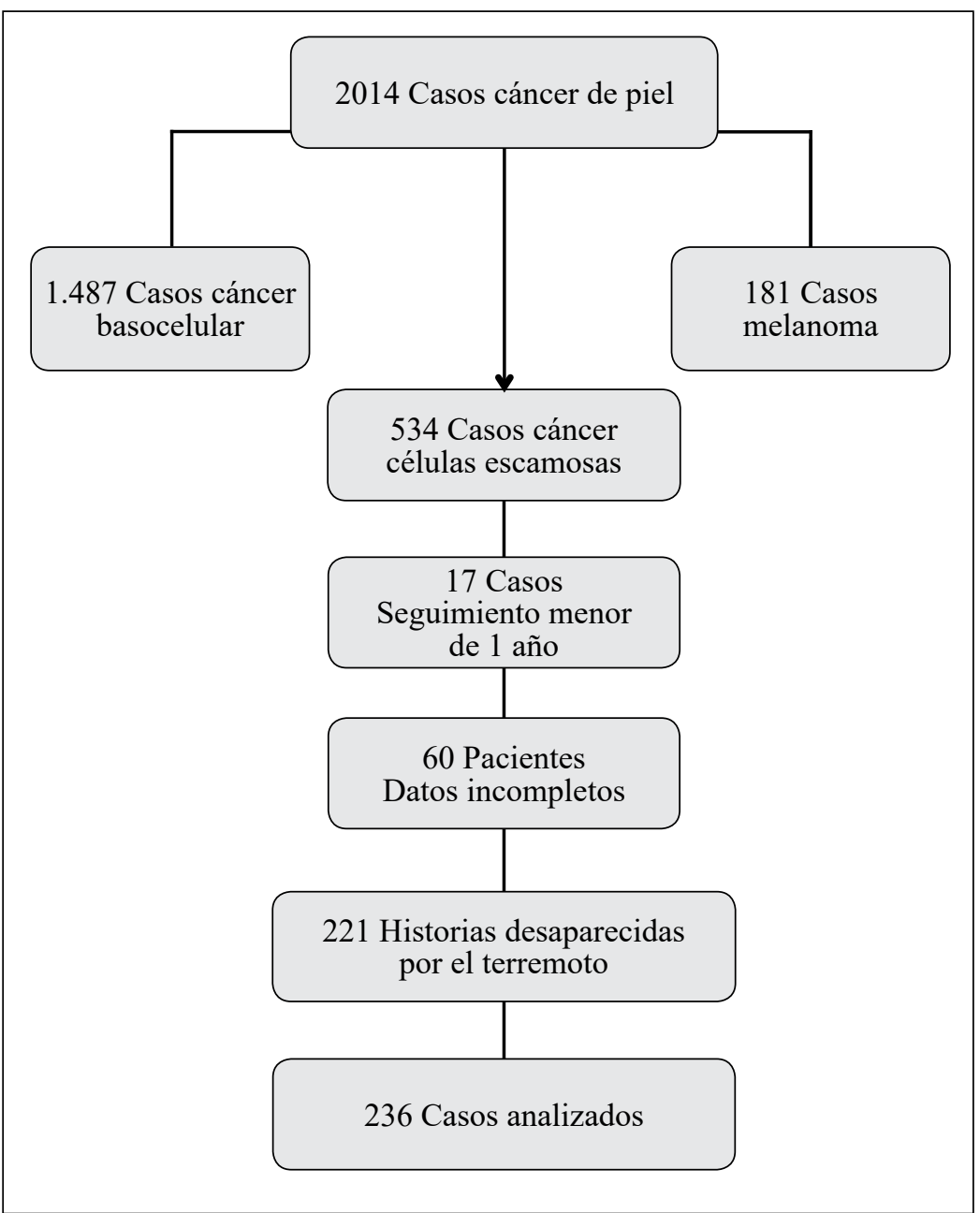

Figura 1. Cáncer de células escamosas de piel. Hospital de Coquimbo 2005-2014.

Tabla 2. Ubicación de las lesiones de cáncer escamoso de piel. Hospital de Coquimbo, años 2005-2014 $(n=236)$

\begin{tabular}{|lrllr|}
\hline Ubicación & $\mathbf{n}(\mathbf{\%})$ & Ubicación en cabeza & n (\%) \\
Cabeza & $158(66,9)$ & Mejilla & $40(16,9)$ \\
Mano & 20 & $(8,5)$ & Frente & $29(12,3)$ \\
Tórax & 20 & $(8,5)$ & Nariz & $22(9,3)$ \\
Pierna & 9 & $(3,8)$ & Oreja & $22(9,3)$ \\
Cuello & 9 & $(3,8)$ & Cuero cabelludo & $20(8,5)$ \\
Antebrazo & 8 & $(3,4)$ & Labio inferior & $14(5,9)$ \\
Abdomen & $5(2,1)$ & Labio superior & $6(2,5)$ \\
Muslo & $3(1,3)$ & Párpado superior & $5(2,1)$ \\
Otros & $4(1,2)$ & Párpado inferior & $2(0,8)$ \\
\hline
\end{tabular}

Tabla 1. Características demográficas de cáncer escamoso de piel. Hospital de Coquimbo, años 2005 a 2014

\begin{tabular}{|c|c|}
\hline Característica & Parámetro \\
\hline Edad & $\begin{array}{l}\text { Promedio } 75 \pm 11,7 \text { años } \\
\text { Rango } 38 \text { a } 94 \text { años }\end{array}$ \\
\hline Sexo & Masculino $64,8 \%(n=153)$ \\
\hline Lugar de residencia & $\begin{array}{l}\text { Costa } 55,5 \%(\mathrm{n}=131) \\
\text { Interior } 44,1 \%(\mathrm{n}=104)\end{array}$ \\
\hline $\begin{array}{l}\text { Exposición de la zona } \\
\text { afectada al sol }\end{array}$ & $\begin{array}{l}\text { Piel expuesta } 87,3 \% \\
(\mathrm{n}=206)\end{array}$ \\
\hline Diámetro & $\begin{array}{l}\text { Promedio } 21,3 \pm 16,4 \mathrm{~mm} \\
\text { Rango } 3 \text { a } 100 \mathrm{~mm}\end{array}$ \\
\hline Fotodaño manifiesto & $84,7 \%(n=200)$ \\
\hline Ulceración & $50,4 \%(\mathrm{n}=119)$ \\
\hline Linfonodos positivos & $4,2 \%(n=10)$ \\
\hline
\end{tabular}

(3,8\%), pierna 9 casos $(3,8 \%)$, antebrazo 9 casos $(3,8 \%)$, abdomen $5(2,1 \%)$, muslo $3(1,3 \%)$ y otras localizaciones 4 casos $(1,6 \%)$. En cabeza el sitio es mejilla 40 casos $(25 \%)$, frente 29 casos $(12,3 \%)$, nariz 22 casos $(9,3 \%)$, oreja 22 casos $(9,3 \%)$, cuero cabelludo 20 casos $(8,5 \%)$, labio inferior 14 casos $(5,9 \%)$, labio superior 6 casos $(2,5 \%)$ y otros en 7 casos $(2,9 \%)$ (Tabla 2$)$.

Hubo 4 casos con linfonodos clínicos y 2 con LNC positivo (4,2\%); se hizo disección de linfonodos simultánea con el tratamiento del tumor primario en 12 casos $(5,1 \%)$, localizados en extremidad superior e inferior. En 12 casos se hizo LNC: los 10 casos de LNC negativo están sanos al cierre del estudio. En tumores de cabeza y cuello no se realizó estudio de LNC. En 230 casos se hizo resección de tejidos blandos $(97,5 \%)$ y en 6 casos se extendió a hueso $(2,5 \%)$.

El cierre del área resecada se hizo en forma primaria en 143 casos $(60,6 \%)$, con injerto dermoepidérmico en 58 casos $(24,6 \%)$, con colgajos simples o musculocutáneos en 33 casos (14\%) y amputación en 2 casos $(0,8 \%)$ En 201 casos los bordes histológicos resultaron libres de tumor $(85,2 \%)$ y en 35 casos el borde está comprometido $(14,8 \%)$ (Tabla 3$)$.

Hubo recurrencia local en 20 casos $(8,5 \%)$ de los cuales 17 tienen borde histológico positivo. No registramos recurrencia en los pacientes con LNC negativo.

El análisis de riesgo de recurrencia muestra asociación con borde de sección histológico positivo, $\mathrm{p}=0,001$, OR 6,17 ; IC $95 \%(3,12-12,19)$ y la presencia de ulceración del tumor, $\mathrm{p}=0,01$, OR 4,63; 
IC 95\% $(1,59-13,45)$. No identificamos asociación entre recurrencia y características histológicas de alto riesgo de recidiva de enfermedad (alto grado histológico, invasión tumoral subcutánea e invasión perineural), $\mathrm{p}=0,05$ OR 0,46; IC 95\% (0,21-1,01). No se identifica asociación entre recurrencia y cáncer primario en áreas no expuestas a radiación UV, $\mathrm{p}=0,21$. No se identificó asociación entre sexo masculino y aparición de recurrencia, $\mathrm{p}=0,66$. Tampoco se identificó asociación entre localización de alto riesgo y recurrencia (oreja, labio) $p=0,37 \mathrm{y}$ tumor de más de $20 \mathrm{~mm}$ de diámetro y recurrencia, $\mathrm{p}=0,36$ (Tabla 4). La letalidad fue de 2,56\% (6 casos).

\section{Discusión}

La región no presenta arsénico inorgánico natural en el agua de consumo humano ${ }^{26,27}$ desde 1980 , por lo cual el riesgo ambiental para la aparición de la enfermedad podemos reducirlo a la exposición a luz ultravioleta, factor de riesgo ya conocido en nuestro medio para cáncer de piel y que es significativamente más alta en los valles interiores ${ }^{18}$.

La frecuencia general de CCE de $25 \%$ es similar a lo reportado en $\mathrm{USA}^{30}$. Nuestra serie comparte las características conocidas de mayor incidencia y prevalencia en adultos mayores, que en nuestra muestra tiene edad media de diagnóstico de 75 años; sin embargo, nuestra incidencia resulta la mitad de otras series ${ }^{15-17}$. Tal diferencia podría generarse por registro de casos parciales porque algunos pacientes se tratan en Centros de otras regiones. En cuatro de cada cinco casos la lesión se ubica en piel expuesta a RUV y con daño por exposición prolongada al sol, confirmando el efecto nocivo de aquella radiación. El riesgo de invasión de linfonodos regionales varía entre 3 y 5\% ${ }^{5-8,28}$. En esta serie el 4,2\% de los casos tenía compromiso ganglionar clínico o microscópico al momento del diagnóstico. A pesar de que la localización en cabeza y cuello es la más frecuente, no realizamos estudio de linfonodo centinela (LNC) en pacientes con cuello porque no contamos con el examen de linfocintigrafía para identificar el primer ganglio de drenaje ${ }^{31}$ y el uso único de azul patente para ello nos resulta inespecífico, por el gran número de ganglios que se tiñen ${ }^{31}$. Por el contrario, en extremidades el LNC nos resulta de fácil acceso solo con el colorante.

Al analizar los factores de recurrencia, el único factor asociado es la presencia de borde histológico positivo para células neoplásicas, es decir, resección insuficiente $\mathrm{y} / \mathrm{o}$ presencia de ulceración. El primero
Tabla 3. Características clínicas y del tratamiento cáncer escamoso de piel. Hospital de Coquimbo (2005-2014)

\begin{tabular}{|llr|}
\hline Característica & & n $(\mathbf{( \% )}$ \\
Linfonodo centinela & No se hizo biopsia & $222(94,1)$ \\
& Negativo & $12(5,1)$ \\
Borde histológico & Positivo & $2(0,8)$ \\
& Negativo & $205(85,2)$ \\
Tipo de resección & Positivo & $35(14,8)$ \\
& Tejido blando & $230(97,5)$ \\
Tipo de cierre & Incluido hueso & $6(2,5)$ \\
& Primario & $143(60,6)$ \\
& Injerto & $58(24,6)$ \\
& Colgajo arterial & $21(8,9)$ \\
& Colgajo al azar & $12(5,1)$ \\
Disección linfonodal & No se hizo & $227(96,2)$ \\
& Simultánea & $9(3,8)$ \\
Radioterapia & No se hizo & $231(97,9)$ \\
& Local & $5(2,1)$ \\
\hline Recurrencia & Libre & $198(83,9)$ \\
& Local & $20(8,5)$ \\
& Ganglionar & $3(1,3)$ \\
& Distante & $(1,3)$ \\
\hline
\end{tabular}

Tabla 4. Factores de recurrencia en 236 casos cáncer escamoso de piel. Hospital de Coquimbo (2005-2014)

\begin{tabular}{|lcc|}
\hline Recurrencia & $\mathbf{n}$ & $\%$ \\
Libre & 198 & 83,9 \\
Local & 20 & 8,5 \\
Ganglionar & 3 & 1,3 \\
Distante & 3 & 1,3 \\
Factores de recurrencia & $\mathbf{p}$ & OR-IC 95\% \\
Borde histológico positivo & 0,001 & $6,17(3,12-12,19)$ \\
Ulceración del tumor & 0,05 & $4,63(1,59-13,45)$ \\
Alto riesgo histólogico & 0,05 & $0,46(0,21-1,01)$ \\
Área expuesta uv & 0,21 & \\
Sexo masculino & 0,66 & \\
Localización alto riesgo & 0,37 & \\
Tumor $>$ 20 mm & 0,36 & \\
Metástasis positivas & 0,56 & \\
Tipo de cierre & 0,37 & \\
\hline
\end{tabular}


ya lo señalamos previamente como el factor más importante para recurrencia en el tratamiento quirúrgico del cáncer basocelular ${ }^{29}$, lo que remarca la necesidad que siempre se realice un estudio de biopsia contemporánea para confirmar la resección completa, como el mejor método para reducir el riesgo de recurrencia. En nuestra serie no encontramos la asociación descrita a localizaciones de alto riesgo y a factores histológicos, probablemente por una muestra reducida.

\section{Responsabilidades éticas}

Protección de personas y animales. Los autores declaran que para esta investigación no se han realizado experimentos en seres humanos ni en animales.

Confidencialidad de los datos. Los autores declaran que han seguido los protocolos de su centro de trabajo sobre la publicación de datos de pacientes.

Derecho a la privacidad y consentimiento informado. Los autores declaran que en este artículo no aparecen datos de pacientes.

\section{Conflicto de intereses}

Los autores no tienen conflicto de intereses que declarar.

\section{Bibliografía}

1. Rudnick EW, Thareja S, Cherpelis B. Oral therapy for nonmelanoma skin cáncer in patientes with advanced disease and large tumor burden: a review of the literatura with focus on a new generation of targeted therapies. Int J Dermatol. 2015 Nov 13. doi: 10.1111/ijd.12961.

2. Metterle L, Nelson C, Patel N. Intralesional 5-fluouracilo (FU) as a treatment for nonmelanoma skin cáncer: A review. J Am Acad Dermatol. 2015 Nov 11: SO190-9622(15)02245-8.

3. Thang ND, Yajima I, Kumasaka MY, Kato M. Bidirectional functions of arsenic as a carcinogen and an anti-cancer agent in human squamous cell carcinoma. PLoS One. 2014 May 9;9(5):e96945. doi: 10.1371/journal.pone.0096945. 2014

4. Liao WT, Yu CL, Lan CC, Lee CH, Chang CH, Chang LW, You HL, Yu SH. Differential effects of arsenic on cutaneous and systemic immunity: focusing on $\mathrm{CD} 4+$ cell apoptosis in patients with arsenic-induced Bowen's disease. Carcinogenesis 2009 Jun;30(6):1064-72. doi: 10.1093/carcin/ bgp095.

5. Natafji N, Tidman MJ. Improving detection of non-melanoma skin cáncer. Practitioner 2015;259(1784):23-7, 3 .

6. Olsen CM, Wilson LF, Green AC, Bain CJ, Fristchi L, Neale RE, et al. Cancers in Australia attributable to exposure to solar ultraviolet radiation and prevented by regular sunscreen use. Aust N Z J Public Health 2015;39:471-6.

7. Dika E, Fanti PA, Misciani C, Vaccari S, Crisman G, Barisani A, et al. Risk of skin cancer development in 672 patients affected by Actinic Keratoses. G Ital Dermatol Venereol. 2016;151:628-33. Epub 2015 Sep 18.

8. Karagas MR, Gossai A, Pierce B, Ahsan H. Drinking Water Arsenic Contamination, Skin Lesions, and Malignancies: A Systematic Review of the Global Evidence. Curr Environ Health Rep. 2015;2:52-68.

9. Kauvar AN, Arpy CJ, Hruza G, Olbricht SM, Bennett R. Consensus for Nonmelanoma Skin Cancer Treatment, Part II: Squamous Cell Carcinoma, Including a Cost Analysis of Treatment Methods. Dermatol Surg. 2015;41:121440.

10. Gore SM, Shaw D, Martin RC, Kelder W, Rorh K, Uren R, et al. Prospective study of sentinel node biopsy for high-risk cutaneous squamous cell carcinoma of the head and neck. Head Neck 2015 May 12. doi: 10.1002/hed.24120. [Epub ahead of print].

11. Gandhi SA, Kampp I.

Skin Cancer Epidemiology, Detection, and Management. Med Clin North Am. 2015;99:1323-35.

12. Ferris RL, Kraus DH. Sentinel lymph node biopsy versus selective neck dissection for detection of metastatic oralsquamous cell carcinoma. Clin Exp
Metastasis 2012;29:693-8.

13. Allen JE, Stollen LB. Utility of sentinel node biopsy in patients with high-risk cutaneous squamous cell carcinoma. Eur J Surg Oncol. 2015;41:197-200.

14. Krediet T, Beyer M, Lenz K, Ulrich C, Lange-Asschemfeldt B, Stochflesh E, et al. Sentinel lymph node biopsy and risk factors for predicting metastasis in cutaneous squamous cell carcinoma. Br J Dermatol. 2015;172:1029-36.

15. Orkic Z, Puntaric D, Puntaric I, Vidosavlievic D, Gvozdic V, Mayer D. Characteristics and incidence of head and neck skin malignant neoplasms in the population of the osijek-baranya county 2004-2012]. Acta Med Croatica 2015;69:15-24.

16. Rudolph C, Schnoor M, Eisenman $\mathrm{N}$, Katalinic A. Incidence trends of nonmelanoma skin cancer in Germany from 1998 to 2010. Deutsch Dermatol Ges. 2015;13:788-97.

17. Nasser N, Nasser Filho N, Lehmukuhl RL. Squamous cell cancer-31-year epidemiological study in a city of south Brazil. An Bras Dermatol. 2015;90:21-6.

18. Iribarren $\mathrm{O}$, Montes $\mathrm{L}$, Barría $\mathrm{C}$, Valle $\mathrm{L}$, Kunkel W. Cáncer Basocelulular en área costera y valles interiores, IV Región. Rev Chil Cir. 2000;52:503-10.

19. Stratigos A, Garbe C, Lebbe C, Malvehy J, Del Marmol V, Pehamberger H, et al. Diagnosis and treatment of invasive squamous cell carcinoma of 
the skin: European consensus-based interdisciplinary guideline. Eur J Cancer 2015;51:1989-2007.

20. Edge S, Compton C. The American Join Comittee on Cancer: the $7^{\mathrm{a}}$ edition of AJCC Cancer Staging Manual and the future of TNM. Ann Surg Oncol. 2010;17:1471-4.

21. Gupta A, Veness M, De'Ambrois B, Selva D, Huilgol SC.

Management of squamous cell and basal cell carcinomas of the head and neck with perineural invasion. Australas J Dermatol. 2015 Mar 11. doi: 10.1111/ ajd.12314.

22. Onajin O, Wetter DA, Roenigk RK, Gibson LE, Eaver AL, Comfere NI. Frozen section diagnosis for nonmelanoma skin cancers: correlation with permanent section diagnosis. J Cutan Pathol. 2015;42:459-64.

23. Iribarren O, Madariaga JA, González
S, Aqueveque C, Rojas M, Saavedra F, et al. Diagnóstico precoz de metástasis de melanoma mediante biopsia de linfonodo centinela. Cuadernos de Cirugía 2007;21:11-16.

24. Seethala RR. Current state of neck dissection in the United States. Head Neck Pathol. 2009;3:238-45.

25. Rogers-Vizena C, Lalonde D, Menick F, Bentz M. Surgical Treatment and Reconstruction of Nonmelanoma Facial Skin Cancers. Plast Reconstr Surg. 2015;135:895e-908e.

26. Laboratorio de salud pública ambiental: Química y microbiología de agua. http:// seremi4.redsalud.gob.cl/wrdprss_minsal/ wpcontent/uploads/2015/05/Listadoanalisis-Laboratorio.pdf.

27. Ahumada G. Tratamiento de agua potable en Chile. Remoción de arsénico. Asociación Interamericana de Ingeniería Sanitaria, 2006. http://www.cedeus.cl/ wp-content/uploads/2014/08/presentaciongahumada-as-uchile-mop-puc.pdf. Consultado el 07 de agosto de 2016.

28. Reszco A, Aasi S, Wilson L. Cancer of the skin. En De Vita, Hellman, Rosemberg: Cancer, Principles \& Practice of Oncology, 2011, 9th Ed Lippincott: 16201624.

29. Iribarren O, San Martín J, Fischer J, Larenas E, Hernández J, Salinas R, et al. Carcinoma basocelular. Factores de recidiva. Rev Chil Cir. 1998;50:420-5.

30. Burton KA, Ashack KA, Khachemoune A. Cutaneous Squamous Cell Carcinoma: A Review of High-Risk and Metastatic Disease. Am J Clin Dermatol. 2016; [Epub ahead of print] Review.

31. Ryu WC, Koh IC, Lee YH, Cha JH, Kim SI, Kim CG. Concordant Surgical Treatment: Non-melanocytic Skin Cancer of the Head and Neck. Arch Craniofac Surg. 2017;18:37-43. 